\title{
Гидродинамические терагерцовые плазмоны и электронный звук в графене с пространственной дисперсией
}

\author{
(C) Д.В. Фатеев ${ }^{1,2}$, В.В. Попов ${ }^{1}$ \\ ${ }^{1}$ Саратовский филиал Института радиотехники и электроники им. В.А. Котельникова \\ Российской академии наук, \\ 410019 Саратов, Россия \\ ${ }^{2}$ Саратовский государственный университет им. Н.Г. Чернышевского, \\ 410012 Саратов, Россия \\ E-mail: FateevDV@yandex.ru
}

Поступила в Редакцию 15 апреля 2020 г.

В окончательной редакции 21 апреля 2020 г.

Принята к публикации 21 апреля 2020 г.

Теоретически исследованы собственные поперечно-магнитные моды графена с пространственной дисперсией в гидродинамическом режиме в терагерцовом частотном диапазоне. Выяснено, что к пространственной дисперсии приводит диффузионный механизм транспорта электронов в графене за счет растекания градиентов электронной концентрации под действием давления в электронной жидкости. Рассмотрены случаи экранированных и неэкранированных плазмонов и электронного звука.

Ключевые слова: плазмон, терагерцовое излучение, графен, пространственная дисперсия, электронный звук.

DOI: $10.21883 /$ FTP.2020.08.49626.02

\section{1. Введение}

Недавние эксперименты продемонстрировали, что транспорт электронов в графене при температурах от азотной до комнатной схож с транспортом вязкой жидкости $[1,2]$. Так, электрический ток может формировать „водовороты“ $[1]$, а проводимость точечных контактов может превосходить предел Шарвина благодаря эффектам вязкого увлечения [2]. Такой гидродинамический транспорт реализуется в условиях сильных электронэлектронных столкновений, частота которых должна являться наибольшей из частот в изучаемой системе. Микроскопические оценки этой частоты дают значение порядка 5 ТГц при комнатной температуре [3]. Отсюда, в частности, следует, что распространение плазмонов терагерцового (ТГц) диапазона в графене должно подчиняться законам гидродинамики. Плазмоны в гидродинамическом режиме могут возбуждаться постоянным током по черенковскому механизму [4], а также трансформироваться в электронно-дырочные звуковые колебания вблизи точки зарядовой нейтральности $[5,6]$. Скорость таких электронно-дырочных звуковых колебаний в графене определяется выражением $v_{\mathrm{F}} / \sqrt{2}$, где $v_{\mathrm{F}}$ - скорость Ферми в графене [4,7]. При замедлении гидродинамических плазмонов до скоростей, близких к скорости Ферми, в графене может возникать пространственная дисперсия проводимости.

Обычно в ТГц-диапазоне при описании проводимости графена используют локальную модель $[8,9]$. Однако полученная в работе [10] проводимость указывает на то, что учет пространственной дисперсии не важен для поперечно-магнитных (ТМ) мод на частотах $<1$ ТГц и, возможно, необходим на более высоких частотах в
ТГц частотном диапазоне при исследовании медленных волн. Тензорная проводимость графена с учетом пространственной дисперсии вычисляется из уравнения Больцмана в $\tau$-приближении Бхатнагара-Гросса-Крука (БГК) [11]. Важность учета пространственной дисперсии при рассмотрении плазмонов в ТГц-диапазоне в графене подчеркивается в работе [12], где тензорная проводимость получена в приближении малых волновых векторов $q$. Показано, что волноводные ТМ-моды в графене при росте частоты в значительной степени определяются пространственной дисперсией. В приближении малых $q$ пространственную дисперсию рассматривают при разложении проводимости, полученной из уравнения Больцмана в $\tau$-приближении, в ряд Тейлора [13].

Учет пространственной дисперсии в графене приводит к уменьшению локализации плазмонов и их затухания в двухслойных графеновых структурах при сильном замедлении плазмонов [14]. В теоретической работе [15] по исследованию распространения плазмонов в периодических структурах получен синий сдвиг частоты плазмонов по сравнению с локальной моделью. Наибольшее влияние пространственной дисперсии возникает при диэлектрическом окружении графена материалами с большими диэлектрическими проницаемостями. Возбуждение плазмонов в дисковых графеновых структурах с пространственной дисперсией теоретически исследовано в работе [16].

Экспериментально влияние пространственной дисперсии было обнаружено в ближнеполевых экспериментах по возбуждению плазмонов [17] как уменьшение их длины волны по сравнению с локальными теоретическими моделями.

В данной работе исследованы собственные ТМ-моды графена с пространственной дисперсией в гидродинами- 
ческом режиме в ТГц частотном диапазоне. Рассмотрены случаи экранированных и неэкранированных плазмонов и электронного звука.

\section{2. Гидродинамическая проводимость графена}

В низкочастотной части ТГц-диапазона динамика носителей заряда в графене описывается гидродинамическими уравнениями $[5,18,19]$ : уравнением непрерывности

$$
\frac{\partial n}{\partial t}+\frac{\partial n v}{\partial x}=0
$$

уравнением баланса импульса носителей заряда

$$
\frac{\partial \rho v}{\partial t}+v \frac{\partial \rho v}{\partial x}+\frac{\partial P}{\partial x}+\frac{\rho}{2} \frac{\partial \rho^{2} v^{2}}{\partial x}+e n E_{x}=-\gamma \rho v
$$

и уравнением баланса энергии

$$
\frac{\partial W}{\partial t}+v_{\mathrm{F}}^{2} \nabla \rho v-e E_{x} j=0
$$

где $n$ и $v-$ соответственно концентрация и гидродинамическая скорость носителей заряда, $\rho-$ плотность массы носителей заряда, $P$ - давление, $e-$ элементарный заряд, $E_{x}$ - компонента электрического поля в плоскости графена, $\gamma$ - частота релаксации импульса носителей заряда в графене, $W=\rho v_{\mathrm{F}}^{2}-P-$ плотность энергии, $v_{\mathrm{F}}=10^{6} \mathrm{M} / \mathrm{c}-$ скорость Ферми носителей заряда в графене и $j=-e n v-$ плотность электрического тока. Давление в графене без постоянного дрейфа носителей заряда может быть вычислено по формуле

$$
P=\frac{\rho v_{\mathrm{F}}^{2}}{3}
$$

В изотропном графене без постоянного дрейфа при малом действующем поле все осциллирующие величины можно представить в следующем виде:

$$
\begin{gathered}
\rho(x, t)=\rho_{0}+\rho_{1} \exp (-i \omega t+i q x), \\
n(x, t)=n_{0}+n_{1} \exp (-i \omega t+i q x), \\
E_{x}(x, t)=E_{1 x} \exp (-i \omega t+i q x), \\
j(x, t)=j_{1} \exp (-i \omega t+i q x), \\
v(x, t)=v_{1} \exp (-i \omega t+i q x), \\
P(x, t)=P_{0}+P_{1} \exp (i \omega t+i q x) .
\end{gathered}
$$

Линеаризация гидродинамических уравнений (1)-(4) в графене без постоянного дрейфа носителей дает систему уравнений

$$
\begin{gathered}
-\omega n_{1}+q n_{0} v_{1}=0, \\
(-i \omega+\gamma) \rho_{0} v_{1}+i q P_{1}=-e E_{1 x} n_{0}, \\
-\frac{2 \omega}{v_{\mathrm{F}}^{2}} P_{1}+q \rho_{0} v_{1}=0, \\
P_{1}=\frac{\rho_{1} v_{\mathrm{F}}^{2}}{3} .
\end{gathered}
$$

Решение линеаризованной системы уравнений (6) дает

$$
\begin{gathered}
v_{1}=-\frac{e n_{0}}{\rho_{0}} \frac{i \omega E_{1 x}}{\left(\omega(\omega+i \gamma)-\frac{q^{2} v_{\mathrm{F}}^{2}}{2}\right)}, \\
n_{1}=\frac{q n_{0}}{\omega} v_{1}, \\
P_{1}=\frac{q \rho_{0} v_{\mathrm{F}}^{2}}{2 \omega} v_{1}, \\
\rho_{1}=\frac{3 P_{1}}{v_{\mathrm{F}}^{2}} .
\end{gathered}
$$

Тогда можно записать закон Ома в фурьепредставленииKD и проводимость $\sigma_{q}$ для изотропного графена без постоянного дрейфа носителей заряда в виде $j_{1}=\sigma_{q} E_{x 1}$, где

$$
\sigma_{q}=\frac{i e^{2} n_{0}}{m} \frac{\omega}{\omega(\omega+i \gamma)-0.5 q^{2} v_{\mathrm{F}}^{2}},
$$

и введена динамическая масса носителей заряда в графене как $m=\rho_{0} / n_{0}$. Слагаемое $0.5 q^{2} v_{\mathrm{F}}^{2}$ в знаменателе проводимости графена, приводящие к пространственной дисперсии, учитывает действие давления в уравнении баланса импульса (2). Проводимость в виде (8) совпадает с проводимостью из [11] в приближении малых $q$, которое верно для волн с фазовой скоростью больше скорости Ферми. Формула, аналогичная (8) встречается в работах $[4,18]$.

Часто при описании плазменных колебаний в двумерном электронном газе действием давления пренебрегают по параметру $v_{\mathrm{pl}} / v_{T}$, где $v_{\mathrm{pl}}-$ фазовая скорость плазмона и $v_{T}$ - диффузионная скорость. В графене это приводит к виду друдевской проводимости

$$
\sigma=\frac{i e^{2} n_{0}}{m(\omega+i \gamma)},
$$

которую можно видеть в работах $[8,9]$ по графеновым плазмонам. В пределе низких температур, когда энергия Ферми электронов больше внутренней энергии $\left(\varepsilon_{\mathrm{F}}>k_{\mathrm{B}} T\right)$, равновесную концентрацию можно вычислить как $n_{0}=\varepsilon_{\mathrm{F}}^{2} /\left(\pi \hbar^{2} \nu_{\mathrm{F}}^{2}\right)$, а динамическую массу как $m=\varepsilon_{\mathrm{F}} / \nu_{\mathrm{F}}^{2}$.

Пространственная дисперсия проводимости графена $\sigma_{q}$ приводит к возникновению точки переключения между емкостным $\left(\operatorname{Im} \sigma_{q}<0\right)$ и индуктивным $\left(\operatorname{Im} \sigma_{q}>0\right)$ режимами проводимости (рис. 1). Условием для этого переключения является равенство нулю знаменателя выражения (8). При этом вблизи точки переключения и действительная и мнимая части проводимости достигают экстремальных величин, а частота точки переключения определяется формулой

$$
\omega=\operatorname{Re}\left[\sqrt{\frac{q^{2} v_{\mathrm{F}}^{2}}{2}-\frac{\gamma^{2}}{4}}-i \frac{\gamma}{2}\right]
$$




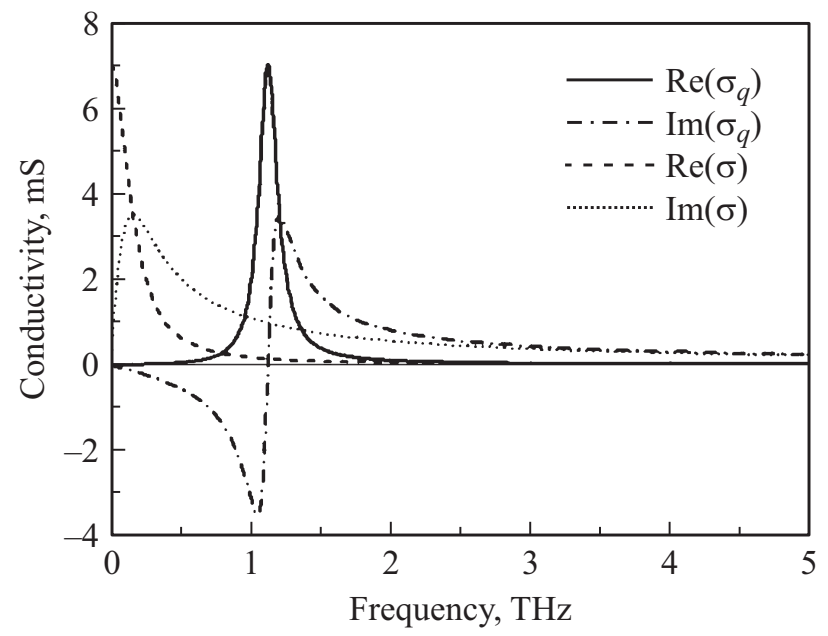

Рис. 1. Спектры действительной (сплошная кривая) и мнимой (штрихпунктирная кривая) частей проводимости с учетом давления в сравнении с действительной (пунктирная кривая) и мнимой (точечная кривая) проводимости без учета давления при $q=10^{7} \mathrm{M}^{-1}, \gamma=10^{12} \mathrm{c}^{-1}, \varepsilon_{\mathrm{F}}=60$ мэВ.

При этом частота (10) - это частота акустической волны в электронной жидкости в отсутствие электрического поля. Скорость такой акустической волны в отсутствие затухания равна $v_{e-\text { sound }}=v_{\mathrm{F}} / \sqrt{2}$. Однако колебания электронов обязательно сопровождаются колебанием электрического поля, и поэтому чистого электронного звука в электронной жидкости быть не может. Свойства волны будут определяться двумя механизмами передачи импульса: кулоновским отталкиванием зарядов и давлением заряженной жидкости.

Поскольку для возбуждения плазмонных резонансов в графене необходима проводимость индуктивного типа, частоты любых собственных плазмонных мод должны лежать выше частоты (10). На частотах гораздо выше частоты переключения (10) (рис. 1) вкладом давления в проводимость можно пренебречь и использовать модель проводимости (9).

\section{3. Плазмонные и электронно-звуковые моды}

Поскольку в области сильной пространственной дисперсии проводимости в ТГц-диапазоне возможно возбуждение плазмонных мод, следует рассмотреть их трансформацию при учете влияния давления в гидродинамических уравнениях.

В квазиэлектростатическом пределе дисперсионное уравнение для неэкранированных плазмонов можно записать в виде [20]

$$
\frac{\varepsilon_{1}}{q}+\frac{\varepsilon_{2}}{q}=-i \frac{\sigma_{q}}{\omega \varepsilon_{0}}
$$

и для экранированных плазмонов как

$$
\frac{\varepsilon_{1}}{q} \operatorname{cth}(q d)+\frac{\varepsilon_{2}}{q}=-i \frac{\sigma_{q}}{\omega \varepsilon_{0}},
$$

где $\varepsilon_{1}$ и $\varepsilon_{2}$ - диэлектрические проницаемости материалов, окружающих графен и $d-$ расстояние от графена до параллельного ему экрана (толщина диэлектрика с диэлектрической проницаемостью $\varepsilon_{1}$ ). Выражения (11) и (12) позволяют получить дисперсионные выражения для собственных мод в графене с учетом пространственной дисперсии

$$
\omega_{P}=\sqrt{\frac{e^{2} \varepsilon_{\mathrm{F}} q}{\pi \hbar^{2} \varepsilon_{0} \tilde{\varepsilon}}-\frac{\gamma^{2}}{4}+\frac{q^{2} \nu_{\mathrm{F}}^{2}}{2}}-\frac{\gamma}{2}
$$

и без учета пространственной дисперсии

$$
\omega=\sqrt{\frac{e^{2} \varepsilon_{\mathrm{F}} q}{\pi \hbar^{2} \varepsilon_{0} \tilde{\varepsilon}}-\frac{\gamma^{2}}{4}}-i \frac{\gamma}{2},
$$

где $\tilde{\varepsilon}=\varepsilon_{1}+\varepsilon_{2}$ - для неэкранированного графена, а $\tilde{\varepsilon}=\varepsilon_{1} \operatorname{cth}(q d)+\varepsilon_{2}$ - для экранированного.

Сравнение дисперсионных кривых с учетом и без учета пространственной дисперсии в неэкранированном графене показывает хорошее совпадение моделей для мод, фазовая скорость которых значительно превышает скорость частиц $v_{\mathrm{F}}$ в графене (рис. 2). Поэтому собственные моды при малых волновых числах представляют собой обычные двумерные плазмоны с корневым законом дисперсии. При росте волновых чисел слагаемое $q^{2} v_{\mathrm{F}}^{2} / 2$ в выражении (13) становится доминирующим

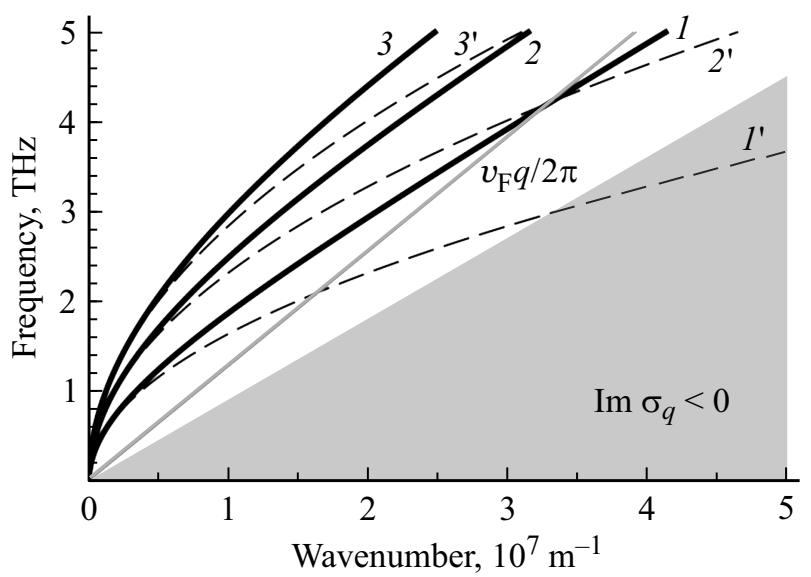

Рис. 2. Дисперсия неэкранированной моды в зависимости от энергии Ферми. Толстые кривые линии 1,2 и 3 - дисперсии, вычисленные в модели с пространственной дисперсией, пунктирные линии $1^{\prime}, 2^{\prime}$ и $3^{\prime}$ вычислены в модели без учета давления. Кривые $1,1^{\prime}$ вычислены при энергии Ферми 20 мэВ, $2,2^{\prime}$ - при 40 мэВ, 3, 3' - при 60 мэВ. Тонкая сплошная линия, соответствующая скорости Ферми в графене, выислена как $f=v_{\mathrm{F}}^{*} q / 2 \pi$. Серая закрашенная область соответствует емкостному типу проводимости графена в модели с пространственной дисперсией. Параметры расчета: $\varepsilon_{1}=\varepsilon_{2}=10$, $\gamma=10^{12} \mathrm{c}$. 


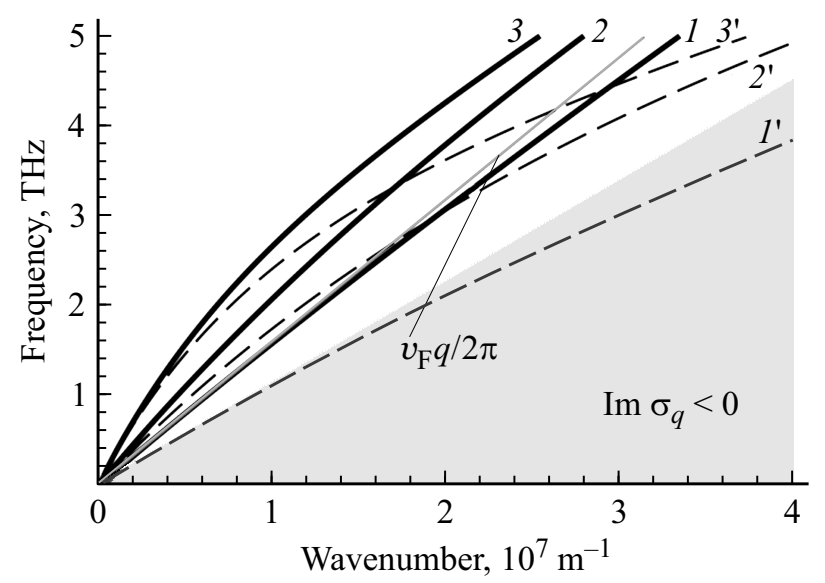

Рис. 3. Дисперсия экранированной моды в зависимости от расстояния до экрана $d$. Толстые кривые линии 1,2 и $3-$ дисперсии, вычисленные в модели с пространственной дисперсией, пунктирные линии $1^{\prime}, 2^{\prime}$ и $3^{\prime}$ вычислены в модели без учета давления. Кривые $1, l^{\prime}$ вычислены при $d=10$ нм, $2,2^{\prime}$ - при 30 нм, 3, 3' - при 100 нм. Тонкая сплошная линия, соответствующая скорости Ферми в графене, вычислена как $f=v_{\mathrm{F}} q / 2 \pi$. Серая закрашенная область соответствует емкостному типу проводимости графена в модели с пространственной дисперсией. Параметры расчета: $\varepsilon_{1}=\varepsilon_{2}=10$, $\varepsilon_{\mathrm{F}}=40 \mathrm{MэB}, \gamma=10^{12} \mathrm{c}^{-1}$.

над кулоновским вкладом $e^{2} \varepsilon_{\mathrm{F}} q /\left(\pi \hbar^{2} \varepsilon_{0}\left(\varepsilon_{1}+\varepsilon_{2}\right)\right)$ и в дисперсионных кривых начинает преобладать линейная зависимость частоты от волнового вектора. Этот факт приводит к трансформации собственных мод в графене от плазмонов к электронному звуку (рис. 2). Типичная зависимость плазмонной дисперсии от энергии Ферми и диэлектрической проницаемости $\omega \propto \sqrt{\varepsilon_{\mathrm{F}} / \varepsilon_{1}}$ в случае c пространственной дисперсией определяет величину вклада кулоновского взаимодействия в механизм передачи импульса в графене (рис. 2). При этом вклад давления в графене неизменен благодаря линейному спектру электронов. Следовательно, все дисперсионные кривые собственных мод в графене лежат в области индуктивной проводимости и асимптотически стремятся к линии электронного звука (10) с ростом волнового числа $q$ (рис. 2). В гидродинамическом режиме существует набор параметров, когда фазовая скорость собственных мод может быть меньше скорости носителей заряда в графене, что может приводить, например, к эффекту Вавилова-Черенкова в графене. Затухание собственных мод и в плазмонном и в электроннозвуковом режимах определяется мнимой частью частоты (13) иравно $\gamma / 2$.

Дисперсионные кривые для экранированных собственных мод в графене при больших волновых числах также стремятся к электронному звуку (10) (рис. 3) и могут быть медленнее носителей заряда. При сильном экранировании кулоновский вклад, как и вклад давления в дисперсию, близок к линейной зависимости частоты от волнового числа, что создает почти линейную дисперсию практически в любом частотном диапазоне (рис. 3).
За счет более сложной зависимости кулоновского вклада экранированых мод от волнового числа $\omega_{P} \propto \sqrt{q /\left(\varepsilon_{1} \operatorname{cth}(q d) \varepsilon_{2}\right)}$ дисперсионные формулы с учетом (13) и без (14) пространственной дисперсии дают значительные отличия в диапазоне малых волновых чисел (рис. 4, $a$ ). Эффект пространственной дисперсии приводит не только к значительному изменению частот собственных мод (рис. 4, $a$ ), но и к уменьшению порога существования волнового решения, возникающего изза релаксации импульса носителей заряда в графене (рис. $4, a$ и $b$ ). Сильная конкуренция между кулоновским и звуковым вкладом в дисперсии происходит по причине экранирования зарядов в графене. Возникающая редукция продольного электрического поля при экранировании приводит к уменьшению энергии возвращающей силы этого поля по сравнению с внутренней энергией электронов в графене. Стоит отметить, что на малых частотах собственные моды в экранированном

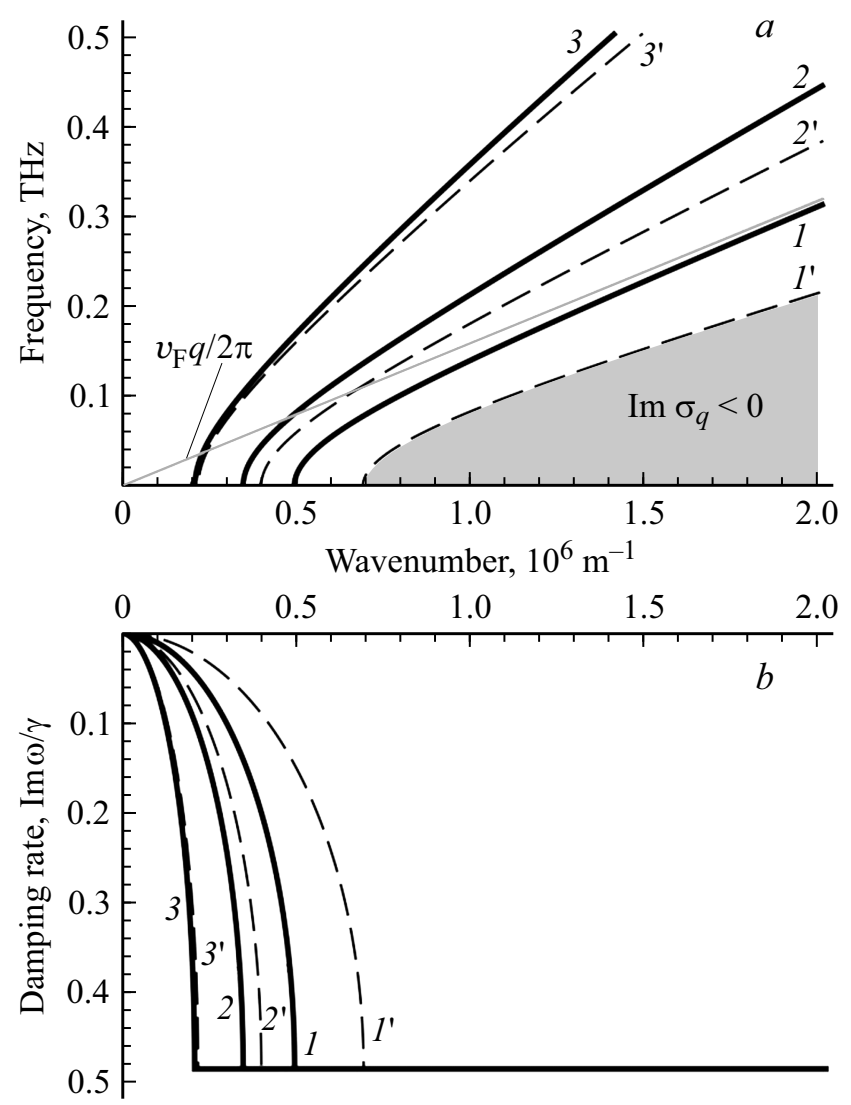

Рис. 4. $a-$ дисперсия экранированной моды при малых волновых числах в зависимости от расстояния до экрана $d$. $b$ - мнимые части частоты в единицах $\gamma$. Толстые кривые линии 1,2 и 3 - дисперсии, вычисленные в модели с пространственной дисперсией, пунктирные линии $1^{\prime}, 2^{\prime}$ и $3^{\prime}-$ вычислены в модели без учета давления. Кривые $1,1^{\prime}$ - вычислены при $d=10$ нм, 2, $2^{\prime}-$ при 30 нм, 3, 3' - при 100 нм. Тонкая сплошная линия, соответствующая скорости Ферми в графене, вычислена как $f=v_{\mathrm{F}} q / 2 \pi$. Серая закрашенная область соответствует емкостному типу проводимости графена в модели с пространственной дисперсией. Параметры расчета: $\varepsilon_{1}=\varepsilon_{2}=10, \varepsilon_{\mathrm{F}}=40 \mathrm{M \ni B,} \gamma=10^{12} \mathrm{c}^{-1}$. 


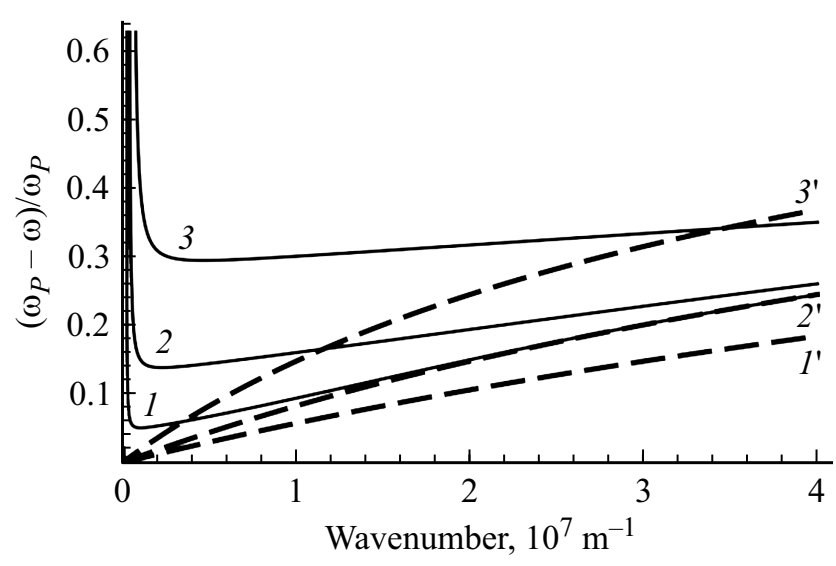

Рис. 5. Относительная разность частот полученными в разных моделях $\left(\omega_{p}-\omega\right) / \omega_{p}$ в зависимости от расстояния до экрана для экранированных плазмонов при энергии Ферми 40 мэВ: сплошные кривые $1-100 \mathrm{Hм}, 2-30 \mathrm{Hм}, 3-10$ нм. Относительная разность частот между моделями в зависимости от энергии Ферми для неэкранированных плазі монов: пунктирные кривые $1^{\prime}-60$ мэВ, $2^{\prime}-40$ мэВ, $3^{\prime}-20$ мэВ. Параметры расчета: $\varepsilon_{1}=\varepsilon_{2}=10, \gamma=10^{12} \mathrm{c}^{-1}$.

графене всегда медленнее носителей заряда. Расчет относительной разности частот $\left(\omega_{P}(q)-\omega(q)\right) / \omega_{P}(q)$ (рис. 5), вычисленных по моделям с учетом (13) и без (14), пространственной дисперсии в графене показывает, что набольшие отклонения между дисперсиями поверхностных мод в моделях с пространственной дисперсией и без нее в графене наблюдаются при бо́льших волновых числах $\left(q>10^{7} \mathrm{M}^{-1}\right)$. В то время как для малых волновых чисел подходит локальная модель проводимости, а собственные моды являются плазмонами. Для сильно экранированных собственных мод во всех диапазонах волновых векторов необходимо учитывать звуковую поправку, однако при малых волновых числах учет пространственной дисперсии понижает порог существования собственных мод.

\section{4. Заключение}

Таким образом, в работе рассмотрено влияние пространственной дисперсии, возникающей за счет учета давления в механизме передачи импульса, на поверхностные электродинамические моды в графене в ТГц-диапазоне частот. Выяснено, что конкуренция кулоновского и звукового вкладов в механизм передачи импульса носителей заряда в графене приводит к изменению дисперсии поверхностных собственных мод в ТГц-диапазоне. Для неэкранированных и экранированных поверхностных мод пространственная дисперсия приводит к трансформации плазмонной моды в электронно-звуковую моду при росте волновых чисел больше $q>10^{7} \mathrm{M}^{-1}$. Для сильно экранированных учет пространственной дисперсии приводит к уменьшению порога возбуждения поверхностных мод, возникающего из-за релаксации импульса носителей заряда.

\section{Финансирование работы}

Работа выполнена в рамках государственного задания и поддержана Российским фондом фундаментальных исследований (№ 18-37-20004) и фондом „БАЗИС“.

\section{Конфликт интересов}

Авторы заявляют, что у них нет конфликта интересов.

\section{Список литературы}

[1] D.A. Bandurin, I. Torre, R. Krishna Kumar, M. Ben Shalom, A. Tomadin, A. Principi, G.H. Auton, E. Khestanova, K.S. Novoselov, I.V. Grigorieva, L.A. Ponomarenko, A.K. Geim, M. Polini. Science, 351, 1055 (2016).

[2] R. Krishna Kumar, D.A. Bandurin, F.M.D. Pellegrino, Y. Cao, A. Principi, H. Guo, G.H. Auton, M. Ben Shalom, L.A. Ponomarenko, G. Falkovich, K. Watanabe, T. Taniguchi, I.V. Grigorieva, L.S. Levitov, M. Polini, A.K. Geim. Nature Phys., 13, 1182 (2017).

[3] D. Svintsov. Phys. Rev. B, 97, 121405(R) (2018).

[4] D. Svintsov. Phys. Rev. B, 100, 195428 (2019).

[5] D. Svintsov, V. Vyurkov, S. Yurchenko, T. Otsuji, V. Ryzhii. J. Appl. Phys., 111, 083715 (2012).

[6] A. Lucas, S. Das Sarma. Phys. Rev. B, 97, 115449 (2018).

[7] A. Lucas. Phys. Rev. B, 93, 245153 (2016).

[8] L.A. Falkovsky, A.A. Varlamov. Eur. Phys. J. B, 56, 281 (2007).

[9] Л.А. Фальковский. УФН, 178 (9), 923 (2008).

[10] G.W. Hanson. IEEE Trans. Antennas and Propagation, 56 (3), 747 (2008).

[11] G. Lovat, G.W. Hanson, R. Araneo, P. Burghignoli. Phys. Rev. B, 87, 115429 (2013).

[12] J.S. Gomez-Diaz, J.R. Mosig, J. Perruisseau-Carrier. IEEE Trans. Antennas and Propagation, 61 (7), 3589 (2013).

[13] P. Li, L.J. Jiang, H. Bağcr. IEEE Trans. Antennas and Propagation, 66 (7), 3590 (2018).

[14] D. Correas-Serrano, J.S. Gomez-Diaz, J. Perruisseau-Carrier, A. Álvarez-Melcón. IEEE Trans. Microwave Theory and Techniques, 61 (12), 4333 (2013).

[15] D. Correas-Serrano, J.S. Gomez-Diaz, A. Alvarez-Melcon. IEEE Antennas and Wireless Propagation Lett., 13, 345 (2014).

[16] D. Mencarelli, S. Bellucci, A. Sindon, L. Pierantoni. J. Phys. D: Appl. Phys., 48, 465104 (2015).

[17] M.B. Lundeberg, Y. Gao, R. Asgari, C. Tan, B. Van Duppen, M. Autore, P. Alonso-González, A. Woessner, K. Watanabe, T. Taniguchi, R. Hillenbrand, J. Hone, M. Polini, F.H.L. Koppens. Science, 357, 187 (2017).

[18] F. Rana. IEEE Trans. Nanotechnology, 7, 91 (2008).

[19] S. Rudin. Int. J. High Speed Electron. Systems, 20 (3), 567 (2011).

[20] M. Jablan, H. Buljan, M. Soljacic. Phys. Rev. B, 80, 245435 (2009).

Редактор Г.А. Оганесян 


\title{
Hydrodynamic terahertz plasmon and electron sound in a graphene with a spatial dispersion
}

\author{
D.V. Fateev ${ }^{1,2}$, V.V. Popov ${ }^{1}$ \\ ${ }^{1}$ Kotelnikov Institute \\ of Radio Engineering and Electronics, \\ Russian Academy of Sciences, \\ 410019 Saratov, Russia \\ ${ }^{2}$ Saratov State University, \\ 410012 Saratov, Russia
}

\begin{abstract}
Transverse-magnetic eigenmodes in graphene with spatial dispersion are theoretically investigated in the hydrodynamic regime in terahertz frequency range. It is shown that the spatial dispersion emerges by the diffusion mechanism of the electron transport as a result of the spreading the electron density gradients under the action of the pressure in electron fluid. Screened and unscreened plasmons as well as electron sound are considered.
\end{abstract}

